American Journal of Applied Sciences 4 (12): 950-956, 2007

ISSN 1546-9239

(C) 2007 Science Publications

\title{
An Investigation on the Effect of the Coupled and Uncoupled Formulation on Transient Seepage by the Finite Element Method
}

\author{
${ }^{1}$ Ahad Ouria, ${ }^{1}$ Mohammad M. Toufigh and ${ }^{1}$ Ali Nakhai \\ ${ }^{1}$ Civil Engineering Department, University of Kerman, Kerman, Iran
}

\begin{abstract}
D transient seepage beneath a dam was investigated by the finite element method. Governing equations were considered in coupled and uncoupled methods. At first stage, the fluid continuity equation for compressible porous media considered as uncoupled. Because of the occurrence of seepage forces, and their interaction with the fluid pore pressure, particularly in high compressible soils, uncoupled methods seem to be far from real conditions. Therefore force equilibrium equations were taken into account by coupling with the fluid continuity equation. Finite element formulation based on Galerkin method. Results of two mentioned methods were compared with steady-state seepage results. Comparing the results of coupled and uncoupled models showed that the time require to reach steady-state condition in coupled model considerably shorter than the uncoupled model. Coupled analyses shows that the effective stress due to seepage forces were smaller than ones calculated by the uncoupled method.
\end{abstract}

Key Words: Transient seepage, couple, uncouple, F.E.M

\section{INTRODUCTION}

Seepage problem is one of the most important issues in the design and construction of dams and hydraulic structures. Seepage forces change stress state, deformation and change permeability of soil elements which would affect the amount of the seepage rate and stability of structure. In routine analyses, steady-state conditions commonly are considered ignoring the time required to reach the steady-state condition. But actually, moving water trough soil voids is a time consuming procedure. Increase of the water table height in a dam reservoir is gradual and time dependent, so transient analysis is essential to yield reliable results.

In addition, in uncouple systems there is only one degree of freedom in the governing equation which is the hydraulic potential. So in order to determine deformations of the structure due to seepage forces it is essential to consider element equilibrium equations besides the fluid continuity equation. In this case a set of partial differential equations including the continuity and equilibrium equations must be solved at the same time.

Considering seepage problem in the coupled form is similar to the coupled consolidation problem except in loading which contains hydraulic potential only. In this research the seepage problem is investigated by two coupled and uncoupled methods. Biot's coupled consolidation equations are adopted to investigate the seepage problem along with the traditional uncoupled transient seepage equation. Galerkin method is used to finite element formulation.

F.E.M formulation for uncoupled system: Fluid flow through porous media is governed by hydrodynamic equations considering the interaction of the fluid in motion with the porous media ensuring the continuity of the fluid. Continuity is ensured by requiring that the net volume of water flowing per unit of time into or out of an element of soil be equal to change per unit of time of the volume of water in that element. Difference of the quantity of the water that leaves or inters to an element is equal to change of the element volume. Therefore the uncoupled fluid continuity equation in the transient state would be as follows ${ }^{[1]}$ :

$$
\frac{\partial v_{x}}{\partial x}+\frac{\partial v_{y}}{\partial y}=-\frac{\partial}{\partial t}(n d v)
$$

Where; $\mathrm{v}_{\mathrm{x}}$ and $\mathrm{v}_{\mathrm{y}}$ are flow velocities in horizontal and vertical directions respectively and, $\mathrm{n}$, is the porosity. The above equation can be rewritten in the following form:

$$
k_{x} \frac{\partial^{2} u_{e}}{\partial x^{2}}+k_{y} \frac{\partial^{2} u_{e}}{\partial y^{2}}=\gamma_{w} m_{v} \frac{\partial u_{e}}{\partial t}
$$

\section{Corresponding Author: Ahad Ouria, Civil Engineering Department, University of Kerman, Kerman, Iran, Phone: $+989143115642$}


Where; $\mathrm{k}_{\mathrm{x}}$ and $\mathrm{k}_{\mathrm{y}}$ are permeability coefficients in horizontal and vertical directions, $\mathrm{u}_{\mathrm{e}}$ is the water pressure and $\mathrm{m}_{\mathrm{v}}$ is the compressibility coefficient of the soil.

Equation 2, is a 2-dimensional consolidation P.D.E which can be solved by the finite element method.

Using linear shape functions with rectangular elements, the final form of the Equation 2 discretized by the F.E.M would be as follows ${ }^{[2]}$ :

$$
K P u_{e}+P M \frac{d u_{e}}{d t}=0
$$

Where;

$K P_{i j}=\iint\left(k_{x} \frac{\partial N_{i}}{\partial x} \cdot \frac{\partial N_{j}}{\partial x}+k_{y} \frac{\partial N_{i}}{\partial y} \cdot \frac{\partial N_{j}}{\partial y}\right) d x \cdot d y$

And:

$$
P M_{i j}=\iint N_{i} N_{j} d x \cdot d y
$$

In the above equations, $\mathrm{N}_{\mathrm{i}}$ is the linear shape function.

Using the Crank-Nicolson's method, time integration of equation 3 involving linear interpolation and fixed time steps $\Delta t$ would yield unconditionally stable and converge results according to Equation $6^{[3]}$ :

$$
\left(P M+\frac{\Delta t}{2} K P\right) u_{e(t+\Delta)}=\left(P M-\frac{\Delta t}{2} K P\right) u_{e(t)}
$$

After finding governing matrix equations for a single element, the assembled matrices for all the elements can be obtained and boundary conditions can be introduced.

Therefore values of unknown variables can be calculated at time $t=t_{0}+\Delta t$ based on known parameters at time $t=t_{0}$.

The initial conditions at time $t=0$ all are known.

F.E.M formulation for coupled system: Biot formulated the theory of coupled solid-fluid interaction where the soil skeleton is treated as porous elastic solid and the laminar pore fluid is coupled to the solid by the conditions of compressibility and of continuity. Thus Biot's governing equations are combination of Equation 1 and element equilibrium equations.

For two-dimensional equilibrium in the absence of body forces considering seepage forces, the gradient of effective stress must be augmented by the gradients of the fluid pressure as follows ${ }^{[2]}$ :

$$
\begin{aligned}
& \frac{\partial \sigma_{x}^{\prime}}{\partial x}+\frac{\partial \tau_{x z}}{\partial y}+\frac{\partial u_{e}}{\partial x}=0 \\
& \frac{\partial \sigma_{y}}{\partial y}+\frac{\partial \tau_{x z}}{\partial x}+\frac{\partial u_{e}}{\partial y}=0
\end{aligned}
$$

The stress-strain relations based on generalized Hook's law for plane strain condition can be written as follows ${ }^{[4]}$ :

$$
\sigma_{i j}=2 G \varepsilon_{i j}+\left(K-\frac{2 G}{3}\right) \delta_{i j}\left(\varepsilon_{11}+\varepsilon_{22}+\varepsilon_{33}\right)(8)
$$

Where, $\mathrm{K}$ and $\mathrm{G}$ are bulk and shear modulus respectively.

Continuity equation can be obtained using Equations 1 and 2 where the volume change of the element is written in terms of displacement components:

$$
\frac{k_{x}}{\gamma_{w}} \cdot \frac{\partial^{2} u_{e}}{\partial x^{2}}+\frac{k_{y}}{\gamma_{w}} \frac{\partial^{2} u_{e}}{\partial y^{2}}=-\frac{d}{d t}\left(\frac{\partial u}{\partial x}+\frac{\partial v}{\partial y}\right)(9)
$$

Where $\mathrm{u}_{\mathrm{e}}$ is defined before, $\mathrm{u}$ and $\mathrm{v}$ are horizontal and vertical components of displacements.

As is usual in the displacement method in solid mechanics, stress and strain are replaced with displacement components so, final coupled variables are the pore water pressure, and horizontal and vertical displacements.

Same as the uncoupled method, using the linear shape functions with rectangular elements for the solid body and the pore fluid, the final form of the set of P.D.E which is combining Equations 7 and 9 discretized by the F.E.M can be as follows ${ }^{[2]}$ :

$$
\begin{aligned}
& K M r+C u_{e}=0 \\
& C^{T} \frac{d r}{d t}-K P u_{e}=0
\end{aligned}
$$

Where: $K P$ is already defined and, $\mathrm{r}$ is the displacement vector $[\mathrm{u}, \mathrm{v}]$; and also $K M$ and $C$ are as follows:

$$
K M=\left[\begin{array}{cc}
K M x x & K M x y \\
K M x y^{T} & K M y y
\end{array}\right]
$$

Where:

$$
\begin{aligned}
& K M x x_{i j}=\iint\left(R_{11} \frac{\partial N_{i}}{\partial x} \frac{\partial N_{j}}{\partial x}+R_{33} \frac{\partial N_{i}}{\partial y} \frac{\partial N_{j}}{\partial y}\right) d x d y \\
& K M x y_{i j}=\iint\left(R_{12} \frac{\partial N_{i}}{\partial x} \frac{\partial N_{j}}{\partial y}+R_{33} \frac{\partial N_{i}}{\partial y} \frac{\partial N_{j}}{\partial x}\right) d x d y( \\
& K M y y_{i j}=\iint\left(R_{22} \frac{\partial N_{i}}{\partial y} \frac{\partial N_{j}}{\partial y}+R_{33} \frac{\partial N_{i}}{\partial x} \frac{\partial N_{j}}{\partial x}\right) d x d y
\end{aligned}
$$


In the above equations, $\mathrm{R}$ is the stress strain relationship matrix according to Equation 8. And also:

$C=\left[\begin{array}{l}C x \\ C y\end{array}\right]$

Where:

$$
\begin{aligned}
C x_{i j} & =\iint N_{i} \frac{\partial N_{j}}{\partial x} d x d y \\
C y_{i j} & =\iint N_{i} \frac{\partial N_{j}}{\partial y} d x d y
\end{aligned}
$$

For integration of the Equation 10 with respect to time, Crank-Nicolson method is implemented, therefore:

$$
\begin{aligned}
& \theta K M r_{1}+\theta C u_{e 1}=(\theta-1) K M r_{0}+(\theta-1) C u_{e 0} \\
& \theta C^{T} r_{1}-\theta^{2} \Delta t K P u_{e 1}=\theta C^{T} r_{0}-\theta(\theta-1) \Delta t K P u_{e 0}(15)
\end{aligned}
$$

In above equations, if $\theta=1$, the system will be absolutely stable without any oscillatory results. Therefore the final form of the Equation 15 in fully implicit type of time-integration will be as follow:

$$
\left[\begin{array}{cc}
K M & C \\
C^{T} & -\frac{\Delta t}{2} K P
\end{array}\right]\left\{\begin{array}{c}
r_{1} \\
u_{e_{1}}
\end{array}\right\}=\left[\begin{array}{cc}
0 & 0 \\
C^{T} & 0
\end{array}\right]\left\{\begin{array}{c}
r_{0} \\
u_{e_{0}}
\end{array}\right\}
$$

After finding governing matrix equations for a single element, the assembled matrices for total elements can be obtained and boundary conditions can be introduced.

Solving such equations at any time, horizontal and vertical deformations $(\mathrm{u}, \mathrm{v})$ and the fluid pressure at various nodal points can be found and strain values for each element can be calculated.

After calculation of primary unknowns, secondary unknowns such as flow fluxes, velocities and effective stresses would be determined.

Calculation of the effective stress in coupled model would be done using the Equation (8) after calculating nodal displacements. In the case of the uncoupled method, because of the absence of displacements, classical formulas are used to determine seepage forces. Vertical and horizontal components of effective stress due to the seepage force would be calculated as ${ }^{[1]}$.

$$
\sigma_{v}^{\prime}=\int_{0}^{z}\left(\gamma_{w} \frac{\partial h}{\partial z}\right) d z
$$

and

$$
\sigma_{h}^{\prime}=\int_{x_{c}}^{x} \gamma_{w} \frac{\partial h}{\partial x} d x,\left.\frac{\partial}{\partial \mathrm{x}}\left(\frac{\partial h}{\partial \mathrm{x}}\right)\right|_{x=x_{c}}=0
$$

In Equation 18, $\mathrm{x}_{\mathrm{c}}$ is the position in which the horizontal gradient of flow has its maximum value. Therefore horizontal seepage force in left and right sides of $x_{c}$ has equal of amount and direction. So the horizontal seepage force in the location of $x=x_{c}$ is zero. Location of $x_{c}$ in each elevation is approximately at the center of the dam and varies slightly. In the left side of $\mathrm{x}_{\mathrm{c}}$ (upstream) the horizontal component of the effective stress is tensile and in the other side is compressive.

\section{RESULTS AND DISCUSSION}

For both coupled and uncoupled formulations, separate finite element codes are developed. In order to compare the effect of the coupled and uncoupled formulations on the seepage calculations, the transient seepage beneath a concrete dam in investigated by both methods. The geometry of the finite element model and material properties are illustrated in Fig.1.

Water table in reservoir rises to 30 meter gradually during 90 days.

At first stage of this study, development of the flow net for coupled and uncoupled analysis is investigated. The resulted equipotential lines for coupled and uncoupled analysis are shown in Figures 2 and 3 respectively.

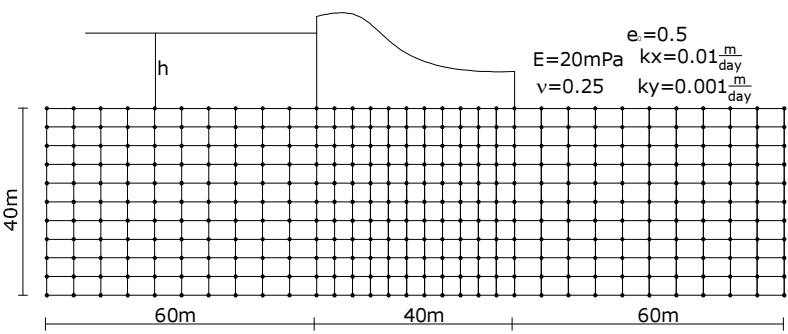

Fig.1: Finite element mesh and material properties 

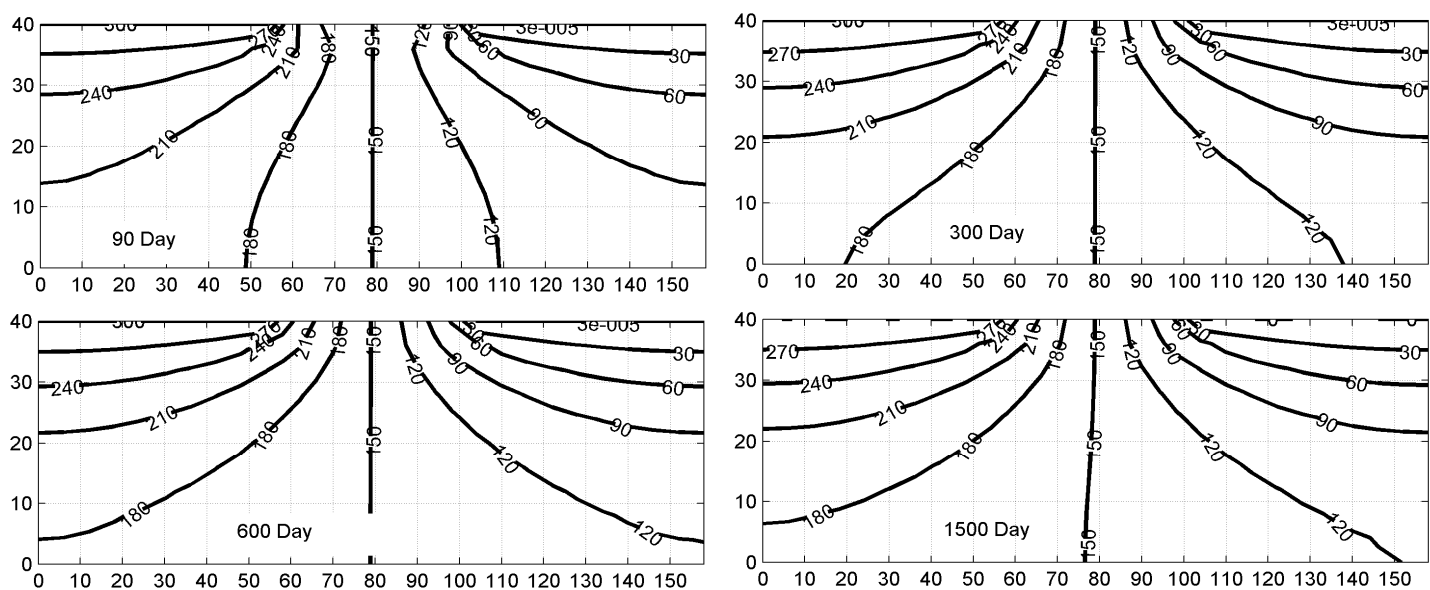

Fig.2: Water pressure distribution resulted from coupled analysis
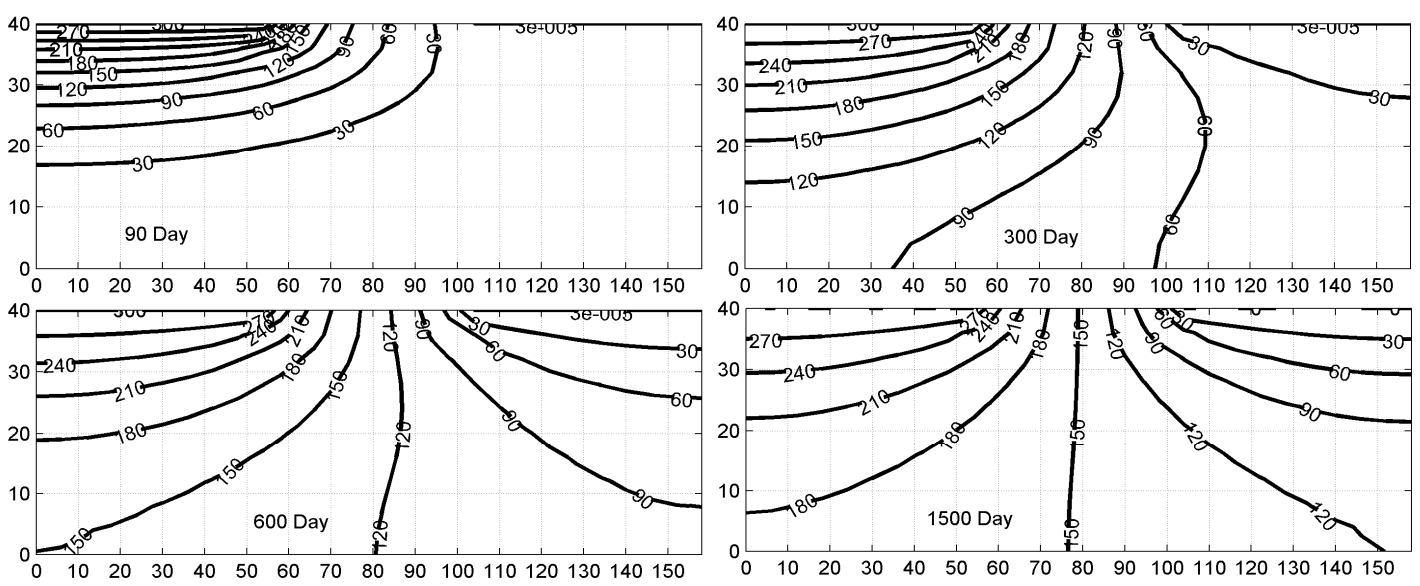

Fig.3: Water pressure distribution resulted from uncoupled analysis

It can be seen from Fig. 2 that the water pressure distribution achieves to steady-state condition after 600 days approximately. Also a symmetrical flow net is observable.

Equipotential lines are moving to the ground surface as the time passes. Increase of the water pressure in down stream is due to seepage forces induced in the upstream and this is because of the coupling effect.

Because the seepage force in the upstream acts as a compressive load and compresses the soil. In the other hand low permeability of the soil skeleton delays the water pressure dissipation process in the down stream and increases the water pressure and also decrease effective stress.

Fig. 3 shows the flow net development beneath the dam resulted from the uncoupled model. Equipotential lines are moving towards down stream as the time passes. It can be seen in Fig. 3 that after 1500 days the steady-state condition is not reached. Also in the case of uncoupled model, unlike the coupled model the flow net is not symmetric until the steady-state condition.

Outlet flow rate from down stream vs. time is illustrated for coupled and uncoupled methods in Fig.4.

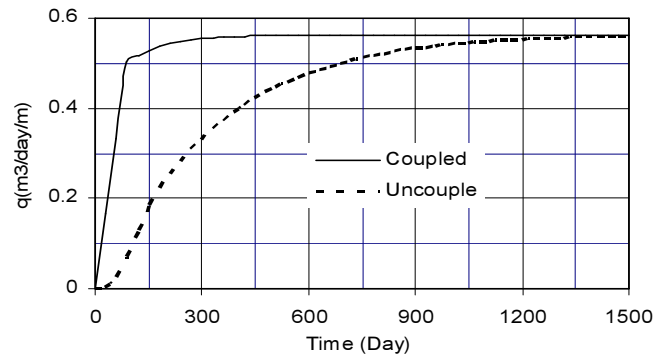

Fig.4: Outlet flow rate for uncoupled and coupled analysis 


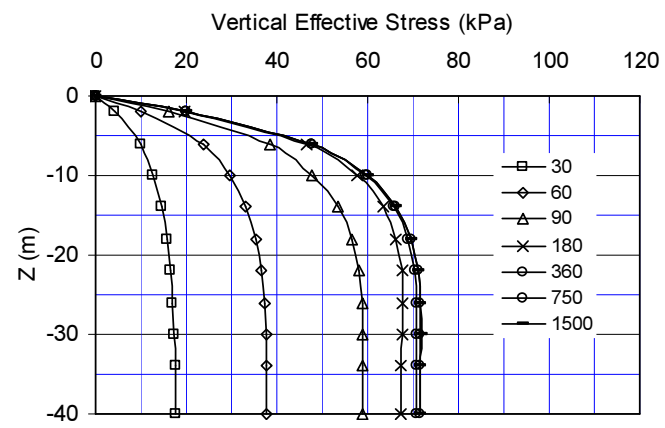

Fig.5: Variation of the vertical effective stress in downstream toe from coupled mode

As mentioned previously for the water pressure distribution in Figures 2 and 3, it can be inferred from Fig.4 that the coupled analysis reaches to steady-state conditions faster than the uncoupled one. Final values of the flow rate for both cases are the same.

In Fig.5, the variation of the effective stress in downstream toe due to seepage forces calculated by the coupled model is plotted in various times.

Effective stress calculated by the uncoupled model based on Equation 17 in the downstream toe is plotted in Fig.6.

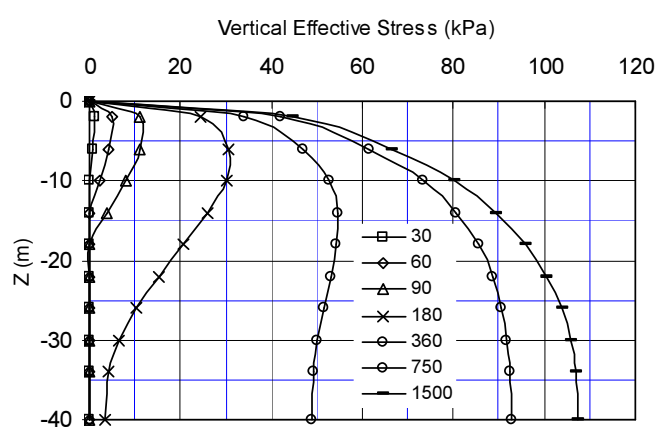

Fig.6: Variation of the vertical effective stress in downstream toe from uncoupled model

Comparing Fig. 5 and Fig. 6 show that the amount of the effective stress calculated based on the seepage gradient is overestimated comparing to the coupled results. It's due to effect of the horizontal effective stress which is omitted in Equation 17.

Uplift pressure beneath the dam in various times, are plotted in Fig. 7 and Fig. 8 for coupled and uncoupled models respectively.

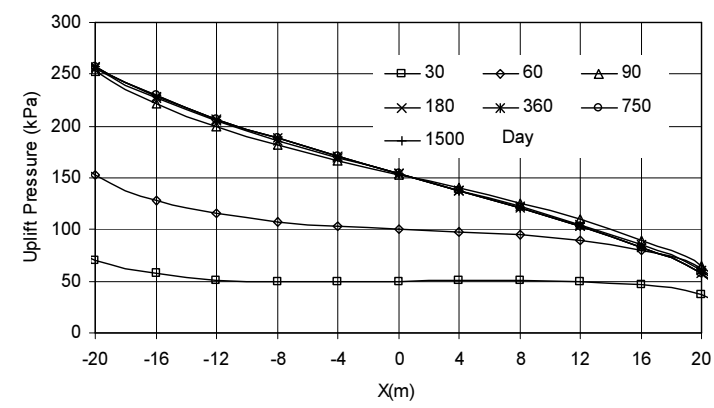

Fig.7: Uplift pressure distribution beneath the dam from coupled model

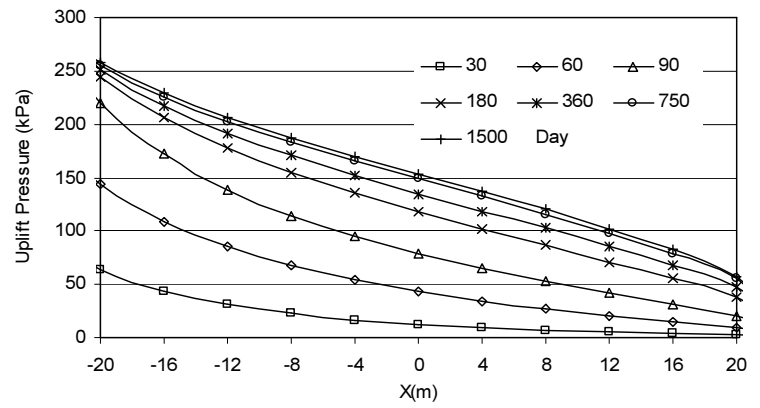

Fig.8: Uplift pressure distribution under dam from uncoupled model

As shown in Figures 7 and 8, uplift pressure results from coupled and uncoupled models are different in amount and distribution in various times but they are equal at steady-state condition. In the coupled model flow net is symmetrical about center of dam but in the case of the uncoupled model it is not symmetrical except at steady-state condition.

In Fig.9, uplift forces for both coupled and uncoupled analyses are shown.

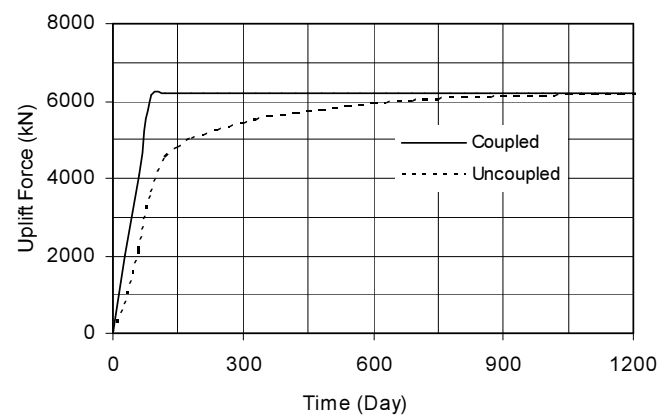

Fig.9: Total uplift force in coupled and uncoupled models 

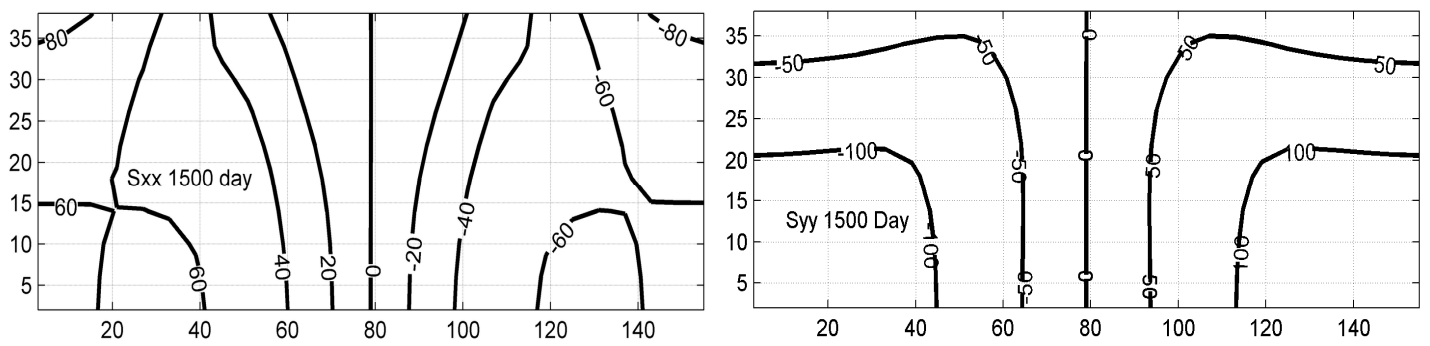

Fig.10: Horizontal and vertical effective stresses due to the seepage force in the coupled model
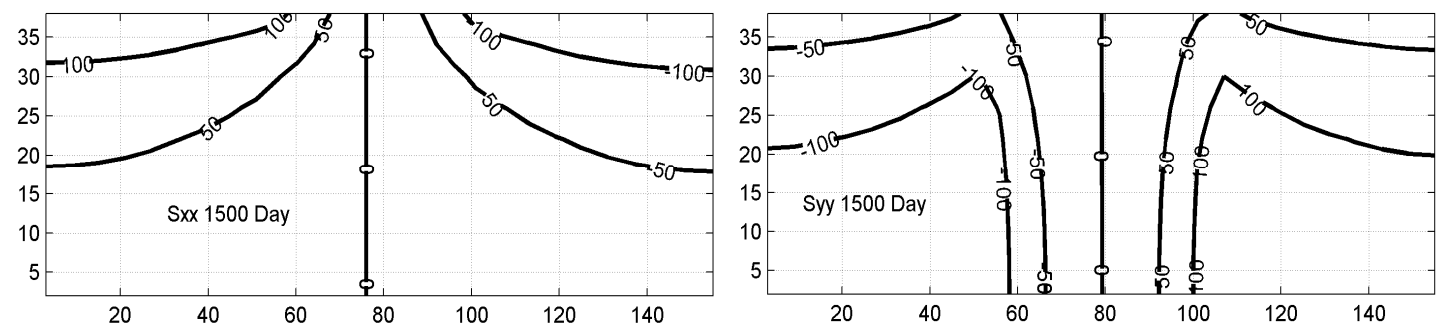

Fig.11: Vertical and horizontal effective stress due to seepage forces form uncoupled model

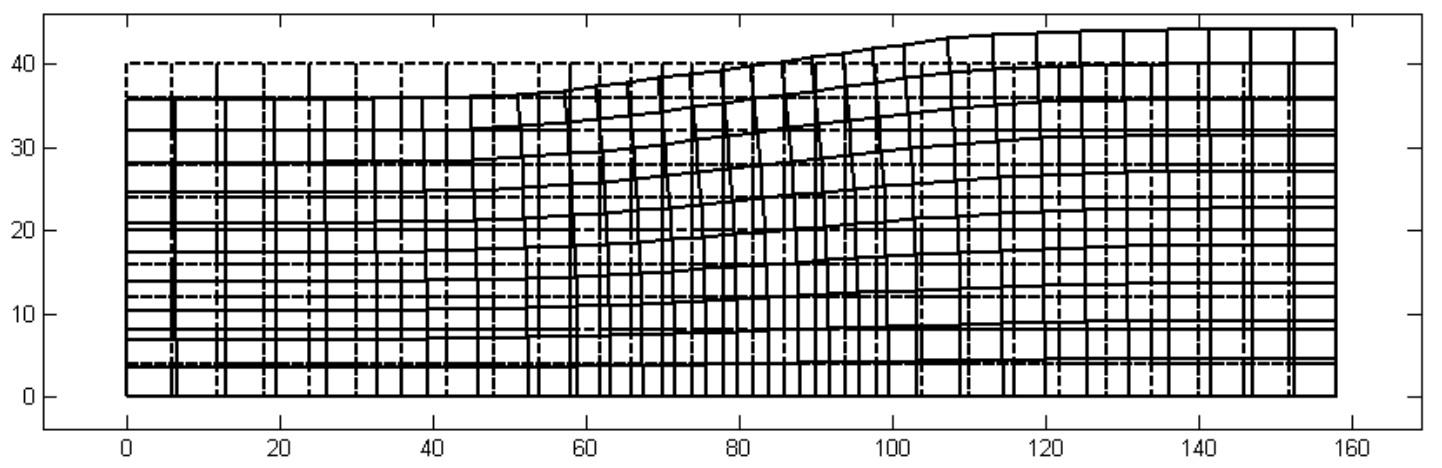

Fig.12: Deformed mesh from coupled model

Vertical and horizontal components of the effective stress due to seepage forces form coupled analyses at steady-state condition are illustrated in Fig. 10.

In Fig. 10, the negative sign refers to compressive stress.

Steady-state effective stress components from uncoupled analysis from Equations 18 and 19 are shown in Fig. 11.

Comparing Figures 10 and 11 shows that the vertical component of effective stress calculated using the coupled and uncoupled models have good correspondences. Values of uncoupled model based on Equation 18 are more than ones resulted from the uncoupled model which is calculated based on calculated displacements. The horizontal effective stress calculated using the Equation 19 widely different from one resulted from coupled model. But its distribution and range have a meaning full relation with the coupled results.

One advantage of the coupled model is its capability of the calculating of the soil deformations due to seepage forces. It would be very important in nonlinear seepage analysis with variable permeability. Also the coupled model would be used to determine additional stresses induced in the dam structure due to its foundation deformations. In the coupled method, nodal displacements are primary unknowns and their values obtain from solutions directly. Fig. 12 shows the deformed mesh due to seepage forces in coupled analyses assuming elastic behavior of soil. In this 
figure, dashed mesh shows initial shape and the displacement of the mesh is magnified 20 times. The maximum displacement of the ground surface is about $0.2 \mathrm{~m}$.

\section{CONCLUSION}

In this paper the effect of the coupled and uncoupled formulations on the results of the confined seepage trough a dam foundation is investigated. The transient state of seepage is due to the change of the water level in reservoir and also porous media compressibility. Compressibility of soil causes the problem to become as a consolidation problem. Therefore uncoupled model is treated as consolidation phenomena. Since the fluid flow produces seepage forces in the soil body and because of the interaction between the seepage forces and the pore fluid pressure, another model based on the Biot's coupled consolidation theory is developed to investigate this interaction. For both cases the finite element technique implemented using the Galerkin method. In the coupled model, the effective stresses are calculated based on the element displacement components which are primary unknowns of the model unlike the uncoupled model where the effective stresses are calculated by the classical formulas of the soil mechanic based on flow gradients in an indirect manner. Therefore the effective stress calculated by the coupled model is more reliable comparing to the uncoupled model.
The results of this study showed that the final values of the outlet flow rate calculated using the coupled and uncoupled methods is equal.

Calculated effective stress by the uncoupled model is grater than the coupled model. Therefore the safety factor of piping in down stream calculated by the uncoupled model is underestimated.

\section{REFERENCES}

1. Karl Terzaghi, Rolph B. Peck, and Golamreza Mesri, 1996. Soil mechanics in engineering practice. 3rd ed, John Wiley, New York, pp 213241

2. Smith I. M., and Griffiths D.V., 2003. Programming the Finite Element. 3rd Ed, John Wiley, pp: 319-440

3. Erik G. Thompson, 2005. Introduction to the Finite Element Method: Theory, Programming and Applications. John Wiley, pp: 150-200

4. S. P. Timoshenko, and J. N. Goodier, 1970. Theory of elasticity. McGraw-Hill, pp: 15-33

5. Toufigh, M. M,. 2002. Seepage with nonlinear permeability by least square F.E.M, Intl J. Engineering., 15(2): 125-134.

6. Toufigh, M. M., and B. Shafiei, 1996. Finite element consolidation model for subsidence problem based on Biot's three dimensional theory. Indian Geotechnical Journal., 26(3): 1-8. 